\title{
Hybrid SEM/AFM System from Carl Zeiss Revolutionizes Analysis of Functional Micro- and Nanostructured Specimen
}

\author{
Nils Anspach ${ }^{1}$, Frank Hitzel ${ }^{1}$, Fang Zhou ${ }^{2}$, and Soeren Eyhusen ${ }^{3}$ \\ 1. DME Nanotechnologie GmbH; Geysostraße 13, 38106 Braunschweig, Germany \\ 2. Carl Zeiss Microscopy, GmbH; Carl-Zeiss-Straße 22, 73447 Oberkochen, Germany \\ 3. Carl Zeiss Microscopy, LLC; 1 Zeiss Dr., Thornwood, NY 10594, USA
}

The Scanning Electron Microscope (SEM) and the Atomic Force Microscope (AFM) are established methods for highest resolution imaging. While the SEM provides a continuous zoom range from millimeter to micrometer dimensions with a very large depth of focus, the AFM provides absolute measurements of all three dimensions down to atomic level, but can only, due to its working principle, measure in the micrometer range. Besides pure topographic information, both methods also provide different kinds of additional information complementing each other, based on their imaging principle. The AFM Option for FESEM from Carl Zeiss provides an integrated solution of both methods [1-3], which overcomes the disadvantages of each method. However, such integration is not always possible due to the magnetic field of the objective lens, the smaller available space under the objective lens and the conventional signal detection methods. Only the unique ZEISS FESEMs [1] which are based on the industry-leading, well-proven, robust and flexible GEMINI technology and the advanced on-axis SE and BSE detection system allow quick and seamless integration with the innovative high-end AFM system made by DME [2].

This combination is the only solution on the market, integrating a full featured laser deflection based AFM in a high-end FESEM, at the same time providing a straight and easy work flow. Tip and sample exchange are performed through the standard load lock, laser alignment for the AFM works fully remote controlled and automatically. The AFM unit is a self contained sample scanner setup with the tip in the eucentric point of the SEM stage tilt axis, see Fig. 1 and [3-4].

The instrument has demonstrated totally new approaches to analyse Helium beam generated nano structures [5], characterize the mechanical stability of suspended Graphene (Fig. 2 and [6]), and imaging surface potential distribution in an active organic solar cell and nano pillars (Fig. 3).

Combining the AFM Option with a crossbeam SEM-FIB (focused ion beam) workstation, in-situ manipulation of tip and sample surface become possible. This has been used by Saive et al. [7-8] to image the electronic work function variation perpendicular to the surface without the need to mechanically break and expose the cross section of a sample. With this tool, local sub-surface investigations by AFM become possible, which are especially important for laterally structured samples.

All these examples are nearly or fully impossible to realize with a standalone AFM together with a standalone SEM. This combination tool opens up a new world of surface inspection tools. It retrieves information from SEM, FIB, and AFM at the same time and from the same spot on the surface, repeatably and routinely. 


\section{References:}

[1] http://microscopy.zeiss.com/microscopy/en_de/products/electron-microscopy/merlinmaterials.html [2] Ahttp: // www.dme-spm.com/ remafm. html

[3] Zeiss Merlin FE-SEM - AFM, http://www.youtube.com/watch?v=0xx8GCcrLPg

[4] Watch the AFM tip at work, http://www.youtube.com/watch?v=yvZIeHfF364

[5] Study of Helium Ion Beam Exposed Nanostructures by in situ AFM, obtainable at [1]

[6] Manipulating Graphene in a hybrid SEM AFM, http://www.youtube.com/watch?v=upaX7t_rh3E

[7] R. Saive et al, Adv. Funct. Mater., 23 (2013), pp. 5854-5860

[8] R. Saive et al., Organic Electronics, 14 (2013), pp. 1570-1576
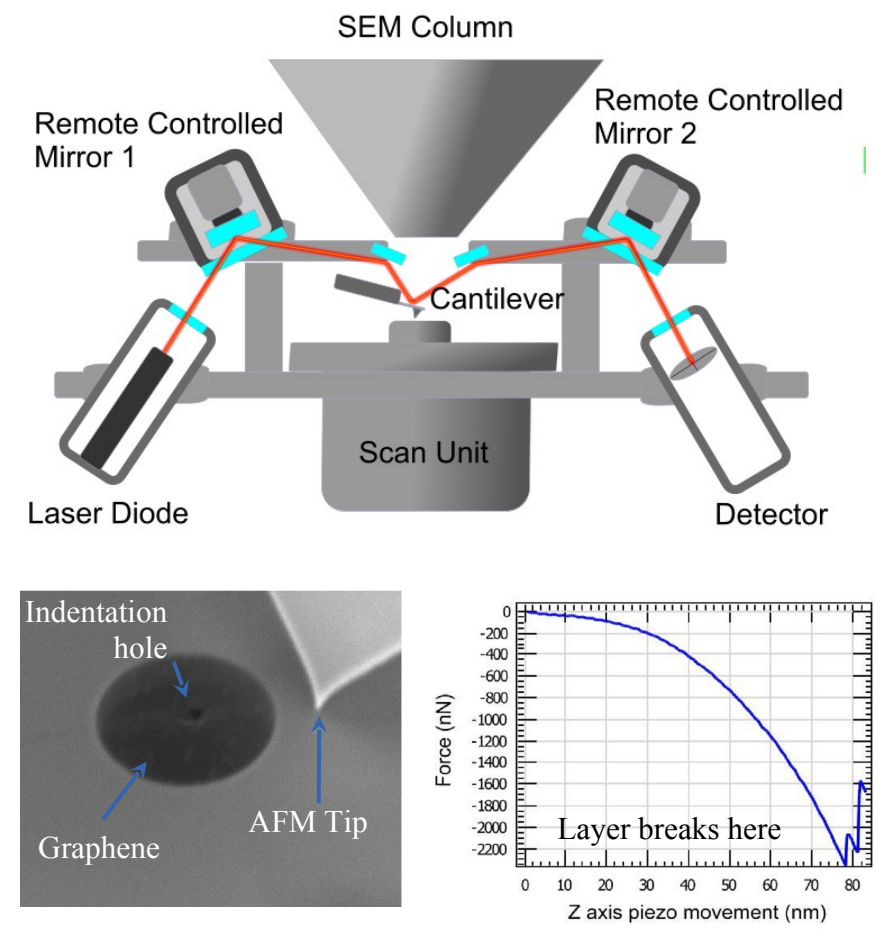

Figure 1. Schematic drawing of AFM Option for FE-SEM scanner part.The system is designed as a sample scanner setup with conventional laser beam path. Short mechanical distances provide a superior mechanical stability down to lateral single atomic resolution inside the running SEM. The arrangement can be tilted to allow viewing angles from 0 to 85 degree.

Figure 2. Mechanical properties of suspended Graphene. The graph shows the mechanical response during indentation, see also [6]. The combination system enables investigating crack propagation and locating Graphene layers with appropriate thickness for the experiment.
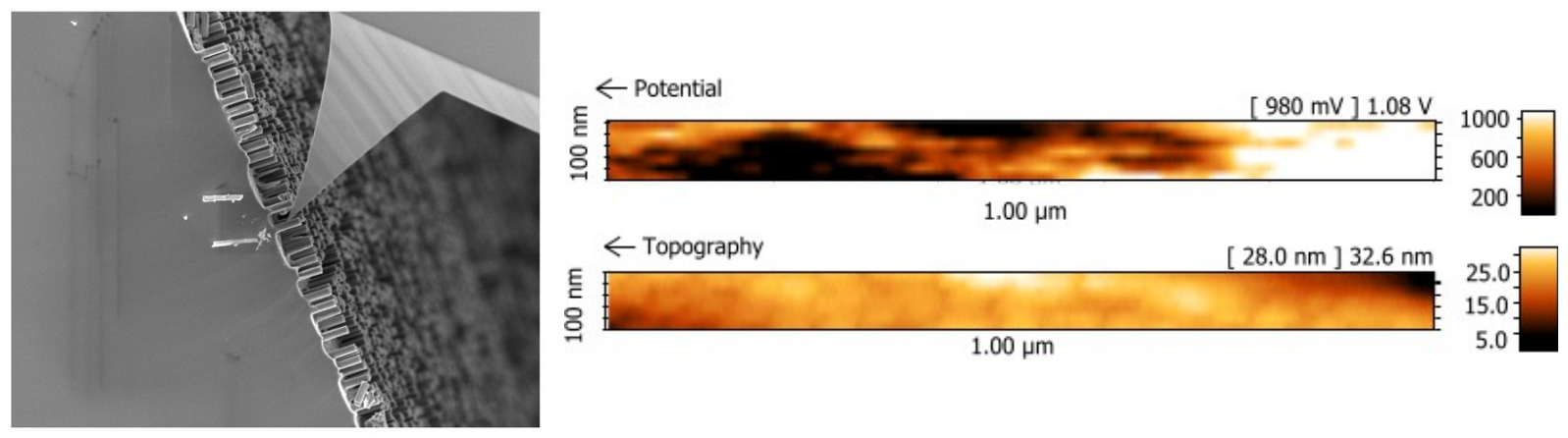

Figure. 3. Analysis of nanopillars by Kelvin Probe Force Microscopy (KPFM). The combined SEM/AFM permits investigation of samples which are totally impossible to work with in a standalone AFM solution. In this example, the work function of one individual FIB-cut nano pillar is investigated. These nano pillars are used for quantum optical experiments. 